\title{
Causas externas em adolescentes: atendimentos em serviços sentinelas de urgência e emergência nas Capitais Brasileiras - 2009
}

\author{
Accidents by external causes in adolescents: care in sentinel urgency \\ and emergency services in the Brazilian State Capitals - 2009
}

\author{
Deborah Carvalho Malta ${ }^{1}$ \\ Márcio Dênis Medeiros Mascarenhas ${ }^{2}$ \\ Regina Tomie Ivata Bernal ${ }^{3}$ \\ Silvania Suely Caribé de Araújo Andrade ${ }^{3}$ \\ Alice Cristina Medeiros das Neves ${ }^{2}$ \\ Elza Machado de Melo ${ }^{4}$ \\ Jarbas Barbosa da Silva Junior ${ }^{1}$
}

${ }^{1}$ Secretaria de Vigiläncia em Saúde, Ministério da Saúde. Esplanada dos Ministérios, Bloco G, Edifício Sede, $1^{\circ}$ andar, Sala 142. 70058-900 Brasília DF.

dcmalta@uol.com.br

${ }^{2}$ Coordenação Geral de

Vigilância de Doenças e

Agravos Não

Transmissíveis

(CGDANT), Secretaria de Vigilância em Saúde (SVS),

Ministério da Saúde (MS).

${ }^{3}$ Faculdade de Saúde

Pública, Universidade de

São Paulo (USP).

${ }^{4}$ Faculdade de Medicina,

Universidade Federal de

Minas Gerais.

\begin{abstract}
Adolescents are seeking new references and experiences, which may involve attitudes of risk and exposure to accidents and violence from external causes. These events constitute a serious Public Health problem. The scope of this study was to analyze the occurrence of accidents by external causes in adolescents from 10 to 19 years of age attended at sentinel urgency and emergency services in Brazil. Data from the 2009 Surveillance System for Violence and Accidents (VIVA 2009) was analyzed in 74 emergency units in 23 state capitals and the Federal District. The findings revealed that 6,434 adolescents (89.8\%) were victims of accidents and 730 (10.2\%) were victims of violence. The main causes of the accidents were falls and traffic accidents, and assaults were predominant in violence. For both accidents and violence, non-white male adolescents were predominant and the events occurred most frequently on the public highways. A marked increase was detected, with hospitalization of victims of violence between 15 and 19 years of age. Understanding the epidemiological reality of external causes among adolescents represents an important tool for health prevention and promotion policies and the culture of peace seeking to reduce morbidity and mortality.
\end{abstract}

Key words Adolescence, External causes, Violence, Accidents, Investigation, Surveillance
Resumo Os adolescentes estão em busca de novas referências e experiências, o que pode implicar atitudes de risco e exposição às causas externas - acidentes e violências. Estes eventos constituem um grave problema em Saúde Pública. O objetivo deste estudo foi analisar as ocorrências de causas externas em adolescentes de 10 a 19 anos atendidos em serviços sentinela de urgência e emergência no Brasil. Foram analisados dados do inquérito da Vigilância de Violências e Acidentes (Inquérito VIVA 2009) em 74 Unidades de Emergência em 23 capitais e no Distrito Federal. Os achados mostraram que 6.434 (89,8\%) adolescentes foram vitimas de acidentes e 730 (10,2\%) de violências. As principais causas de acidentes foram as quedas, outros acidentes e o trânsito e entre as violências predominaram as agressões. Tanto para acidentes como para violências os adolescentes do sexo masculino e a raça/cor não branca foram predominantes; as ocorrências foram mais frequentes na via pública. A alta foi a evolução mais frequente, com maior ocorrência de internação hospitalar nas vítimas de violências entre 15 a 19 anos. Conhecer a realidade epidemiológica das causas externas entre os adolescentes representa uma importante ferramenta para as politicas de prevenção, promoção à saúde e da cultura de paz, visando à redução da morbimortalidade.

Palavras-chave Adolescência, Causas externas, Violências, Acidentes, Inquérito, Vigilância 


\section{Introdução}

A adolescência, período compreendido entre as idades de 10 a 19 anos $^{1}$, tem sido considerada um grupo estratégico para as políticas de promoção à saúde e prevenção de doenças e agra$\operatorname{vos}^{2}$. Nesta fase da vida, os adolescentes estão em busca de novas referências e experiências, o que por vezes pode implicar atitudes de risco e exposição às causas externas - acidentes e violências. Estes eventos podem resultar em lesões e incapacidades definitivas, causando danos físicos, emocionais, sociais, além de intenso sofrimento para as famílias e gastos com assistência à saúde ${ }^{3-5}$, constituindo-se em grande problema em Saúde Pública para a maioria dos países ${ }^{2-4}$.

As causas externas constituem importantes fatores de morbimortalidade na adolescência; no Brasil, em 2011, elas foram responsáveis por 128.316 internações de pacientes entre 10 a 19 anos, em hospitais que integram o Sistema Único de Saúde, e por 16.050 óbitos $^{6}$. As quedas, os acidentes de transporte (AT) e os choques contra objetos inanimados são os tipos mais prevalentes de causas externas entre crianças e adolescentes, acometendo, em maior proporção, o sexo masculino $^{7-10}$.

A Organização Mundial da Saúde define a violência como o uso da força física ou do poder real ou em ameaça, contra si próprio, contra outra pessoa, ou contra um grupo ou uma comunidade, que resulte ou tenha qualquer possibilidade de resultar em lesão, morte, dano psicológico, deficiência de desenvolvimento ou privação ${ }^{3}$. A violência é um fenômeno multicausal que apresenta forte associação com desigualdades econômicas e socioculturais, mas também se relaciona com aspectos subjetivos e comportamentais vigentes em cada sociedade ${ }^{5,11,12}$.

No Brasil, a violência assumiu grande importância pela sua magnitude, gravidade, impacto social e capacidade de vulnerabilizar as vítimas e suas famílias. As crianças, os adolescentes e os jovens estão entre os grupos populacionais mais vitimizados pela violência ${ }^{6}$. Diversos são os tipos de violências que envolvem crianças e adolescentes na sociedade, dentre eles, a intrafamiliar, que envolve toda ação e/ou omissão cujo efeito é o comprometimento do bem-estar, da integridade física e psicológica e da liberdade e/ou direito de desenvolvimento de membro da família. Segundo Minayo ${ }^{13}$, esta condição implica uma transgressão no poder/dever de proteção do adulto e da sociedade em geral e, na [...] negação do direito que crianças e adolescentes têm de serem trata- dos como sujeitos e pessoas em condições especiais de desenvolvimento.

Autores descrevem que entre crianças mais jovens predomina a violência intrafamiliar, como negligência, maus-tratos e/ou violência sexual ${ }^{14} \mathrm{e}$ entre adolescentes a violência física (agressões) tende a ser mais frequente, em função do contexto urbano de violência, desigualdades, exposição a brigas, envolvimento com armas, disputas de gangues e exposição a atos violentos praticados por desconhecidos, consumo de álcool e outras drogas nas vias públicas, escolas, dentre outros ${ }^{5,12,15}$.

Vários são os fatores que contribuem para que essa prática seja observada e mantida, dentre os quais destacamos: as relações de poder e de gênero predominantes nas sociedades, a exclusão social em suas múltiplas formas de expressão, as características do agressor e da vítima, questões culturais, medo de denunciar, ineficiência dos órgãos de atendimento, certeza de impunidade, dentre outras. Tudo isso, apesar da promulgação do Estatuto da Criança e do Adolescente (ECA), segundo o qual qualquer ato de violência praticado contra adolescentes deve ser compreendido como uma violação dos Direitos Humanos ${ }^{16}$.

Os acidentes e as violências na adolescência envolvem peculiaridades marcantes em relação à faixa etária, ao local de ocorrência e às características ou circunstâncias em que se desenvolvem. $\mathrm{Na}$ medida em que aumentam os estudos sobre acidentes e violências, identificando os fatores e os processos da sua ocorrência, as circunstâncias e o ambiente social em que ocorrem, essa realidade tende a mudar, ou pelo menos, a tornar-se mais visível e, desta forma, a evoluir no sentido de redução da impunidade e de proteção das vítimas.

Além disto, os estudos sobre esta temática contribuem para esclarecer as circunstâncias, provendo mais informações; desta forma, apóiam o desenho de políticas públicas de prevenção e promoção à saúde e cultura de paz ${ }^{17}$. Em 2006, o Ministério da Saúde implantou o Sistema de Vigilância de Violências e Acidentes (VIVA), que está organizado em dois componentes: a) contínuo (integra o Sistema de Informação de Agravos de Notificação por meio da notificação universal feita pelos serviços de saúde do país) e, b) inquérito (estudo transversal em serviços selecionados de urgência e emergência no período de trinta dias a cada dois anos), este último será objeto de análise no atual trabalho ${ }^{18}$.

O presente artigo tem por objetivo analisar dados do VIVA inquérito, por acidentes e violências, em adolescentes de 10 a 19 anos, atendidos 
em serviços sentinela de urgência e emergência no Brasil, segundo os desfechos e as circunstâncias do atendimento.

\section{Métodos}

Foram analisados dados do Inquérito VIVA 2009, realizado em 74 Unidades de Emergência em 23 capitais e no Distrito Federal. A metodologia de seleção dos estabelecimentos está descrita em outros trabalhos ${ }^{18}$. O tamanho da amostra foi de, no mínimo, 1.500 atendimentos por causas externas (acidentes e violências) em cada uma das capitais e no Distrito Federal, assumindo coeficiente de variação inferior a 30\% e o erro padrão menor que três. Foi utilizado processo de amostragem por conglomerado em único estágio, sendo o turno a unidade primária de amostragem (UPA). O inquérito envolvia todas as faixas etárias, entretanto para fins do presente estudo foram analisadas apenas as ocorrências na faixa etária de 10 a 19 anos, totalizando 7.164 adolescentes.

Para a composição da amostra foram relacionados todos os serviços conveniados ao SUS e habilitados para atendimento de urgência e emergência no Cadastro Nacional de Estabelecimentos de Saúde (CNES) e selecionados os estabelecimentos que apresentaram maior proporção de atendimentos no Sistema de Informação Hospitalar (SIH/SUS) e no VIVA 2006 e 2007 em cada capital. Procedeu-se à validação da lista de serviços junto aos gestores de cada município, visando ajustar aos critérios de maior demanda da população, percebida pelos gestores. Maiores detalhes da amostra podem ser vistos em outra publicação ${ }^{18}$.

Todos os atendimentos por causas externas do turno sorteado foram incluídos na amostra. Para o sorteio de turnos considerou-se o período de coleta de 30 dias consecutivos divididos em dois turnos, totalizando 60 turnos passíveis de coleta, sendo 30 diurnos e 30 noturnos. $\mathrm{O}$ número de turnos a ser sorteado em cada estabelecimento foi obtido em função do tamanho mínimo da amostra de atendimentos por causas externas no mesmo estabelecimento registrado pelo inquérito VIVA em pesquisas ocorridas em anos anteriores, 2006 e $2007^{18}$.

Os coordenadores de campo dos estados e municípios foram capacitados pela equipe do Ministério da Saúde para a coleta de dados em cada cidade. As entrevistas foram realizadas usando-se um questionário padronizado e testado em inquéritos prévios, o informante era a vítima ou o acompanhante, quando o paciente era menor ou encontrava-se impossibilitado de responder ${ }^{18}$. O projeto foi aprovado pela Comissão Nacional de Ética em Pesquisa (CONEP), atendendo às recomendações da Resolução 196/1996 ${ }^{19}$.

Como os dados são utilizados para fins de vigilância epidemiológica, a assinatura do termo de consentimento livre e esclarecido foi substituída por consentimento verbal, obtido pelo paciente ou por seu responsável. Foi garantido total anonimato e privacidade aos pacientes, profissionais e gestores dos serviços onde a pesquisa foi realizada, assim como a liberdade em desistir de participar da entrevista a qualquer momento, sem prejuízo de qualquer natureza para si próprio ou terceiros.

Os dados foram digitados nos municípios e transferidos para o Ministério da Saúde para análise de consistência e duplicidade, utilizando o programa Link Plus, versão 2.0. As análises foram processadas no programa STATA versão 10.0, do qual se utilizou o módulo "svy", adequado para obtenção de estimativas dos parâmetros populacionais não viciadas quando os dados são provenientes de planos de amostragem complexos. No presente estudo foram selecionados os registros de atendimentos de adolescentes de 10 a 19 anos e analisadas as seguintes variáveis: sexo, faixa etária, raça/cor, meios de locomoção para chegar ao serviço de urgência, local de ocorrência, natureza da lesão, parte do corpo atingida, evolução na emergência.

No caso das vítimas de acidentes foram analisadas as variáveis referentes ao tipo de acidente (acidente de transporte - AT, quedas, queimadura, outros acidentes); entre os AT identificou-se o tipo de vítima (pedestre, condutor e passageiro) e o meio de locomoção no momento do acidente (a pé, automóvel, motocicleta, bicicleta, transporte coletivo). Também foram descritos os tipos de quedas, queimaduras e demais causas acidentais. Para as violências foram avaliados: o tipo (autoprovocada ou agressão) e se o agressor era familiar ou não. As variáveis foram comparadas segundo a sua ocorrência e analisadas segundo duas faixas etárias (10 a 14 anos, 15 a 19 anos) testando-se a hipótese nula de igualdade, utilizando-se o teste qui-quadrado, com nível de significância de $5 \%$.

\section{Resultados}

Foram analisadas informações referentes a 7.164 adolescentes de 10 a 19 anos captados no Inquéri- 
to VIVA 2009. Dentre os participantes, 6.434 $(89,8 \%)$ foram vítimas de acidentes e 730 (10,2 $\%)$ de violências. As características dos adolescentes atendidos nos serviços de emergência, segundo o tipo de ocorrência estão apresentadas na Tabela 1. Em relação à idade, entre 10 a 14 anos predominam as ocorrências por acidentes, e entre 15 a 19 anos foram mais frequentes ocorrências por violência; sendo significativa a diferença ( $\mathrm{p}$
$<0,05)$ entre as duas faixas etárias estudadas. Os adolescentes do sexo masculino predominaram entre as vítimas, sem diferença significativa entre os tipos de ocorrência. Quanto à variável raça, $67,5 \%$ eram não brancos (pardos, pretos, amarelos e indígenas), com predomínio de pardos e pretos (dados não mostrados). A diferença na proporção de não brancos foi significativamente maior nas vítimas de violência $(\mathrm{p}<0,05)$.

Tabela 1. Distribuição dos atendimentos de emergência por acidentes e violência entre adolescentes, segundo as variáveis do estudo e tipo de ocorrência, em 23 capitais e Distrito Federal, Brasil, 2009.

\begin{tabular}{|c|c|c|c|c|}
\hline \multirow[b]{2}{*}{ Características } & \multicolumn{3}{|c|}{ Tipo de ocorrência ${ }^{a}$} & \multirow[b]{2}{*}{$\begin{array}{c}\text { Valor de } \\
\mathrm{p}^{\mathrm{b}}\end{array}$} \\
\hline & $\begin{array}{c}\text { Total } \\
(\mathrm{n}=7164) \\
\%\end{array}$ & $\begin{array}{c}\text { Acidentes } \\
(\mathrm{n}=6434) \\
\%\end{array}$ & $\begin{array}{c}\text { Violência } \\
(\mathbf{n}=730) \\
\%\end{array}$ & \\
\hline \multicolumn{5}{|l|}{ Faixa etária (anos) } \\
\hline 10 a 14 & 48,3 & 50,8 & 25,2 & \multirow[t]{2}{*}{0,0000} \\
\hline 15 a 19 & 51,7 & 49,3 & 74,8 & \\
\hline \multicolumn{5}{|l|}{ Sexo } \\
\hline Masculino & 65,7 & 65,6 & 66,5 & \multirow[t]{2}{*}{0,6580} \\
\hline Feminino & 34,3 & 34,4 & 33,5 & \\
\hline \multicolumn{5}{|l|}{ Raça/cor da pele } \\
\hline Branca & 32,5 & 33,3 & 24,7 & \multirow{2}{*}{0,0001} \\
\hline Não branca & 67,5 & 66,7 & 75,3 & \\
\hline \multicolumn{5}{|l|}{ Locomoção para o hospital } \\
\hline A pé/Transporte coletivo & 27,0 & 27,7 & 20,2 & \multirow[t]{4}{*}{0,0000} \\
\hline Veículo particular & 48,6 & 49,4 & 40,9 & \\
\hline Assistência pré-hospitalar ${ }^{c}$ & 21,7 & 21,1 & 27,4 & \\
\hline Outros $^{\mathrm{d}}$ & 2,7 & 1,8 & 11,5 & \\
\hline \multicolumn{5}{|l|}{ Local de ocorrência } \\
\hline Domicílio & 29,6 & 29,4 & 31,5 & \multirow[t]{4}{*}{0,0000} \\
\hline Escola/Prática esportiva & 24,9 & 26,3 & 11,5 & \\
\hline Via pública & 36,5 & 35,7 & 44,6 & \\
\hline Outros $^{\mathrm{e}}$ & 9,0 & 8,7 & 12,4 & \\
\hline \multicolumn{5}{|l|}{ Natureza da lesão } \\
\hline Contusão/entorse/luxação & 43,7 & 45,7 & 23,8 & \multirow[t]{4}{*}{0,0000} \\
\hline Corte/laceração/amputação & 29,7 & 27,6 & 49,4 & \\
\hline Fratura/trauma ${ }^{\mathrm{f}}$ & 20,6 & 21,2 & 14,9 & \\
\hline Outros & 6,1 & 5,5 & 12,0 & \\
\hline \multicolumn{5}{|l|}{ Parte do corpo atingida } \\
\hline Cabeça/pescoço & 17,9 & 16,0 & 36,8 & \multirow[t]{5}{*}{0,0000} \\
\hline Tórax/abdome/pélvis & 5,7 & 4,7 & 14,9 & \\
\hline Membros superiores & 35,8 & 37,3 & 21,4 & \\
\hline Membros inferiores & 33,4 & 35,8 & 9,4 & \\
\hline Múltiplos órgãos/regiões & 7,2 & 6,2 & 17,5 & \\
\hline \multicolumn{5}{|l|}{ Evoluçãog } \\
\hline Alta & 81,8 & 82,8 & 72,0 & \multirow[t]{3}{*}{0,0000} \\
\hline Internação hospitalar & 8,1 & 7,3 & 15,1 & \\
\hline Encaminhamento para outro serviço & 10,2 & 9,9 & 12,9 & \\
\hline
\end{tabular}

Fonte: MS, SVS, Sistema de Vigilância de Violências e Acidentes-VIVA, Inquérito 2009.

a) Para algumas variáveis o número de atendimentos divergiu devido a dados faltantes (ignorado/em branco); b) Teste do qui-quadrado; c) Inclui unidades do Serviço de Atendimento Móvel de Urgência (SAMU), ambulâncias, resgate; d) Inclui viatura policial e outros; e) Inclui bar/similar, comércio/serviços, indústria/construção, outros; f) Inclui trauma cranioencefálico, trauma dentário, politraumatismo; g) Excluídos os casos que evoluíram ao óbito ( $\mathrm{n}=14 ; 0,2 \%)$ nas primeiras 24 horas de atendimento. 
Os modos de locomoção mais frequentemente utilizados para chegar ao hospital foram veículo particular, a pé/transporte coletivo, ambos com maior proporção entre as vítimas de acidentes. A remoção pelo atendimento pré-hospitalar (SAMU), que aparece em terceiro lugar, foi maior entre adolescentes vítimas de violência ( $\mathrm{p}<0,05)$. A via pública foi o local com maior percentual de ocorrências, seguida do domicílio; nos casos de violência, a via pública foi o local mais referido $(p<0,05)$. Em relação à natureza da lesão, predominaram as contusões/entorses nos atendimentos decorrentes de acidentes e os cortes/laceração, naqueles em consequência das violências. Os membros superiores foram os segmentos corporais mais frequentemente atingidos nos acidentes e a cabeça/pescoço, nas violências $(\mathrm{p}<0,05)$. A alta foi o desfecho mais prevalente, em $82,8 \%$ dos casos de acidentes e em $72 \%$ daqueles relacionados às violências; a internação ocorreu em $15,1 \%$ dos atendimentos por violências $(\mathrm{p}<0,05)$ (Tabela 1$)$.

Na Tabela 2, são apresentadas as distribuições dos acidentes e violências segundo faixa etá-

Tabela 2. Distribuição dos atendimentos de emergência por acidentes e violência entre adolescentes, segundo variáveis de estudo, tipo de ocorrência e faixa etária, em 23 capitais e Distrito Federal, Brasil, 2009.

\begin{tabular}{|c|c|c|c|c|c|c|c|c|}
\hline \multirow[b]{2}{*}{ Características } & \multicolumn{3}{|c|}{ Acidentes $^{\mathrm{a}}$} & \multirow[b]{2}{*}{$\begin{array}{l}\text { Valor } \\
\text { de } p^{b}\end{array}$} & \multicolumn{3}{|c|}{ Violência $^{a}$} & \multirow[b]{2}{*}{$\begin{array}{l}\text { Valor } \\
\text { de } p^{b}\end{array}$} \\
\hline & $\begin{array}{c}\text { Total } \\
\begin{array}{c}(\mathrm{n}=6434) \\
\%\end{array}\end{array}$ & $\begin{array}{c}10 \text { a } 14 \\
(n=3297) \\
\%\end{array}$ & $\begin{array}{c}15 \text { a } 19 \\
(n=3137) \\
\%\end{array}$ & & $\begin{array}{c}\text { Total } \\
(\mathbf{n}=730) \\
\%\end{array}$ & $\begin{array}{c}10 \text { a } 14 \\
(n=183) \\
\%\end{array}$ & $\begin{array}{c}15 \text { a } 19 \\
(n=547) \\
\%\end{array}$ & \\
\hline \multicolumn{9}{|l|}{ Sexo } \\
\hline Masculino & 65,6 & 66,1 & 65,1 & \multirow{2}{*}{0,4614} & 66,5 & 57,4 & 69,6 & \multirow{2}{*}{0,0109} \\
\hline Feminino & 34,4 & 33,9 & 34,9 & & 33,5 & 42,6 & 30,4 & \\
\hline \multicolumn{9}{|l|}{ Raça/cor da pele } \\
\hline Branca & 33,3 & 33,0 & 33,5 & \multirow[t]{2}{*}{0,7091} & 24,7 & 28,3 & 23,6 & \multirow[t]{2}{*}{0,2547} \\
\hline Não branca & 66,7 & 67,0 & 66,5 & & 75,3 & 71,7 & 76,4 & \\
\hline \multicolumn{9}{|l|}{ Locomoção para o hospital } \\
\hline A pé/Transporte coletivo & 27,7 & 30,6 & 24,8 & \multirow[t]{4}{*}{0,0000} & 20,2 & 29,5 & 17,1 & \multirow[t]{4}{*}{0,0000} \\
\hline Veículo particular & 49,4 & 50,5 & 48,2 & & 40,9 & 50,0 & 37,9 & \\
\hline Assistência pré-hospitalar ${ }^{c}$ & 21,1 & 17,5 & 24,9 & & 27,4 & 16,0 & 31,2 & \\
\hline Outros $^{\mathrm{d}}$ & 1,8 & 1,5 & 2,1 & & 11,5 & 4,5 & 13,8 & \\
\hline \multicolumn{9}{|l|}{ Local de ocorrência } \\
\hline Domicílio & 29,4 & 33,1 & 25,5 & \multirow[t]{4}{*}{0,0000} & 31,5 & 32,8 & 31,1 & \multirow[t]{4}{*}{0,0000} \\
\hline Escola/Prática esportiva & 26,3 & 32,8 & 19,5 & & 11,5 & 25,2 & 7,0 & \\
\hline Via pública & 35,7 & 29,5 & 42,1 & & 44,6 & 34,9 & 47,8 & \\
\hline Outros $^{\mathrm{e}}$ & 8,7 & 4,6 & 12,8 & & 12,4 & 7,1 & 14,2 & \\
\hline \multicolumn{9}{|l|}{ Natureza da lesão } \\
\hline Contusão/entorse/ luxação & 45,7 & 47,9 & 43,4 & \multirow[t]{4}{*}{0,0003} & 23,8 & 33,2 & 21,0 & \multirow[t]{4}{*}{0,0195} \\
\hline Corte/laceração/amputação & 27,6 & 25,4 & 30,0 & & 49,4 & 37,7 & 52,8 & \\
\hline Fratura/trauma ${ }^{\mathrm{f}}$ & 21,2 & 21,9 & 20,4 & & 14,9 & 16,5 & 14,4 & \\
\hline Outros & 5,5 & 4,7 & 6,2 & & 12,0 & 12,6 & 11,8 & \\
\hline \multicolumn{9}{|l|}{ Parte do corpo atingida } \\
\hline Cabeça/pescoço & 16,0 & 16,0 & 16,0 & \multirow[t]{5}{*}{0,0000} & 36,8 & 36,0 & 37,1 & \multirow[t]{5}{*}{0,3485} \\
\hline Tórax/abdome/pélvis & 4,7 & 3,6 & 5,9 & & 14,9 & 12,9 & 15,5 & \\
\hline Membros superiores & 37,3 & 41,7 & 32,7 & & 21,4 & 26,4 & 19,8 & \\
\hline Membros inferiores & 35,8 & 34,8 & 36,9 & & 9,4 & 11,3 & 8,8 & \\
\hline Múltiplos órgãos/regiões & 6,2 & 3,9 & 8,4 & & 17,5 & 13,5 & 18,8 & \\
\hline \multicolumn{9}{|l|}{ Evoluçãog } \\
\hline Alta & 82,8 & 84,6 & 80,9 & \multirow[t]{3}{*}{0,0014} & 72,0 & 75,3 & 70,9 & \multirow[t]{3}{*}{0,0002} \\
\hline Internação hospitalar & 7,3 & 6,1 & 8,5 & & 15,1 & 5,6 & 18,3 & \\
\hline Encaminhamento para outro serviço & 9,9 & 9,2 & 10,6 & & 12,9 & 19,1 & 10,8 & \\
\hline
\end{tabular}

Fonte: MS, SVS, Sistema de Vigilância de Violências e Acidentes-VIVA, Inquérito 2009.

a) Para algumas variáveis o número de atendimentos divergiu devido a dados faltantes (ignorado/em branco); b) Teste do qui-quadrado; c) Inclui unidades do Serviço de Atendimento Móvel de Urgência (SAMU), ambulâncias, resgate; d) Inclui viatura policial e outros; e) Inclui bar/similar, comércio/serviços, indústria/construção, outros; f) Inclui trauma cranioencefálico, trauma dentário, politraumatismo; g) Excluídos os casos que evoluíram ao óbito $(\mathrm{n}=14 ; 0,2 \%)$ nas primeiras 24 horas de atendimento. 
ria (10 a 14 e 15 a 19 anos). Não houve diferença estatisticamente significativa quanto ao sexo entre as faixas etárias nos acidentes. Nos casos de violências foi maior a participação masculina entre os adolescentes mais velhos (15 a 19 anos) (p $<0,05)$. Em relação à raça/cor também não houve diferença significativa entre as faixas etárias. Nos casos de acidentes e violências, o modo de locomoção para o hospital mais frequente foi o veículo particular em ambas as faixas etárias, todavia foram observadas diferenças significativas entre as faixas etárias quanto ao modo de locomoção tanto para os acidentes quanto para as violências $(\mathrm{p}<0,05)$.

Quanto ao local de ocorrência para acidentes predominou a via pública entre os adolescentes de 15 a 19 anos, seguido do domicílio, e entre aqueles com 10 a 14 anos foi maior a proporção de acidentes no domicílio $(\mathrm{p}<0,05)$. Dentre os pacientes atendidos devido a violências prevaleceram a via pública nos adolescentes de ambas as faixas etárias, seguido do domicílio. Todavia, há diferença entre os locais de ocorrência entre os dois grupos de adolescentes quanto ao local de ocorrência da situação de violência $(\mathrm{p}<0,05)$. Salienta-se maior percentual de adolescentes mais novos $(25,2 \%)$ comparado com $7,0 \%$ dos mais velhos que apresentam como local de ocorrência da violência a prática esportiva/escola que é o terceiro lugar em ambas as faixas etárias (Tabela 2).

Dentre os atendimentos por acidentes, a natureza das lesões apresentou diferenças entre as faixas etárias $(p<0,05)$; lesões do tipo contusão/ entorse/luxação foram as mais frequentes entre os adolescentes das duas faixas etárias analisadas. Os membros superiores foram a parte do corpo mais atingida entre os adolescentes de $10 \mathrm{a}$ 14 anos que sofreram acidentes (41,7\%) e os membros inferiores foram acometidos em 36,9\% dos adolescentes acidentados de 15 a 19 anos. Lesões em múltiplos órgãos foram mais frequentes em 15 a 19 anos (8,4\%), denotando a gravidade do acidente. Foram constatadas diferenças entre as faixas etárias para a parte do corpo mais atingida nos atendimentos por acidentes $(\mathrm{p}<0,05)$. Os cortes foram as lesões mais referidas entre os adolescentes vítimas de violências, porém, houve diferença estatisticamente significativa quanto à natureza da lesão entre as faixas etárias $(\mathrm{p}<0,05)$. A cabeça foi a parte do corpo mais atingida, sem diferença entre as faixas etárias. (Tabela 2).

Verificaram-se diferenças significativas $(\mathrm{p}<$ $0,05)$ entre as faixas etárias para a evolução nas primeiras 24 horas de atendimento tanto para as vítimas de acidentes quanto para aquelas de vio- lências: a alta foi o desfecho mais frequente nos indivíduos mais novos tanto para acidentes quanto violência. Ressalta-se que $18,3 \%$ dos casos de violência entre os adolescentes de 15 a 19 anos evoluíram para internação (Tabela 2).

A Tabela 3 detalha os atendimentos segundo tipo de evento e faixa etária das vítimas. Dentre os eventos acidentais, predominaram as quedas $(36,9 \%)$, sendo mais frequentes entre os adolescentes de 10 a 14 anos $(44,5 \%)$ e o segundo entre os de 15 a 19 anos. Houve diferença estatística entre as faixas etárias e os tipos de acidentes $(\mathrm{p}<$ $0,05)$. Predominaram as quedas do mesmo nível em ambas as faixas etárias, em seguida as quedas de andaime se destacaram entre adolescentes com 15 a 19 anos (15,8\%) e quedas em buraco/ outros níveis entre os adolescentes de 10 a 14 anos $(13,1 \%)$, revelando diferença significativa entre as faixas etárias $(\mathrm{p}<0,05)$.

O terceiro grupo de eventos mais frequente foi o acidente de transporte (AT) (23,5\%) predominando em adolescentes de 15 a 19 anos (32,6\%), entre os quais representaram o primeiro lugar em ocorrência. O tipo de vítima por AT mais frequente foi o condutor $(59,7 \%)$, tanto para os jovens de 15 a 19 anos (60,1\%) quanto para aqueles de 10 a 14 anos de idade (58,8\%). Verificou-se que a proporção de pedestres vítimas de AT foi significativamente maior entre os adolescentes de 10 a 14 anos $(19,2 \%)$ comparados aos de 15 a 19 anos $(10,3 \%)$ $(\mathrm{p}<0,05)$. O meio de locomoção da vítima no momento do AT foi a motocicleta $(44,3 \%)$, com destaque para o grupo de 15 a 19 anos (56,9\%), enquanto a bicicleta foi o veículo mais frequente entre os AT envolvendo os adolescentes de 10 a 14 anos $(51,6 \%)$. Essas diferenças foram significativas entre os dois grupos etários $(\mathrm{p}<0,05)$.

As queimaduras representaram $1,8 \%$ dos eventos acidentais, sendo mais frequentes as devidas a substâncias quentes $(37,1 \%)$, seguida daquelas decorrentes de contato com fogo/chama (21,8\%), sem diferença entre as faixas etárias. Dentre os outros acidentes, a segunda causa de atendimento, destacam-se o choque contra objetos/pessoa $(33,2 \%)$, que predominam entre os adolescentes de 10 a 14 anos, os ferimentos cortantes $(19,6 \%)$, os entorses e esmagamentos $(19,6 \%)$, estes últimos prevalentes entre os indivíduos de 15 a 19 anos. Foram observadas diferenças significativas entre as faixas etárias quanto ao tipo de outros acidentes $(\mathrm{p}<0,05)$ (Tabela 3$)$.

$\mathrm{O}$ tipo de violência mais comum foi a agressão (90,3\%); a proporção de lesões autoprovocadas foi de 9,7\%, sem diferença entre as faixas etárias. O agressor era membro da família em 
$12,3 \%$ dos casos, característica esta predominante na faixa etária de 10 a 14 anos $(18,2 \%)$ comparada com os maiores de 15 anos ( $<<0,05)$.

Quanto ao dia de ocorrência dos eventos captados no Inquérito VIVA 2009, a maioria dos AT e das agressões ocorreram nos finais de semana, as quedas e outros acidentes predominaram nos dias de semana (Figura 1). Quanto ao horário, os AT tiveram pico de ocorrência por volta das 20 horas $(\mathrm{h})$, seguido das $12 \mathrm{~h}$ às $14 \mathrm{~h}$ e também

Tabela 3. Distribuição dos atendimentos de emergência por acidentes e violência entre adolescentes, segundo características do evento e faixa etária, em 23 capitais e Distrito Federal, Brasil, 2009.

\begin{tabular}{|c|c|c|c|c|}
\hline \multirow[b]{2}{*}{ Características } & \multicolumn{3}{|c|}{ 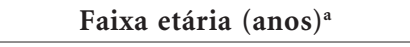 } & \multirow[b]{2}{*}{$\begin{array}{c}\text { Valor de } \\
\mathrm{p}^{\mathrm{b}}\end{array}$} \\
\hline & $\begin{array}{c}\text { Total } \\
(\mathbf{n}) \\
\% \\
\end{array}$ & $\begin{array}{c}10 \text { a } 14 \\
(\mathrm{n}) \\
\%\end{array}$ & $\begin{array}{c}15 \text { a } 19 \\
\text { (n) } \\
\%\end{array}$ & \\
\hline Tipo de acidente & $(6434)$ & $(3297)$ & $(3137)$ & \\
\hline Acidente de transporte & 23,5 & 14,7 & 32,6 & 0,0000 \\
\hline Queda & 36,9 & 44,5 & 29,0 & \\
\hline Queimadura & 1,8 & 1,3 & 2,3 & \\
\hline Outros acidentes & 37,8 & 39,5 & 36,0 & \\
\hline Acidente de transporte: tipo de vítima & $(1595)$ & $(501)$ & $(1094)$ & \\
\hline Pedestre & 13,1 & 19,2 & 10,3 & 0,0001 \\
\hline Condutor & 59,7 & 58,8 & 60,1 & \\
\hline Passageiro & 27,2 & 22,0 & 29,6 & \\
\hline Acidente de transporte: meio de locomoção da vítima & $(1589)$ & $(497)$ & $(1092)$ & \\
\hline A pé & 12,7 & 18,7 & 9,9 & 0,0000 \\
\hline Automóvel & 7,4 & 6,3 & 7,9 & \\
\hline Motocicleta & 44,3 & 17,2 & 56,9 & \\
\hline Bicicleta & 30,3 & 51,6 & 20,4 & \\
\hline Transporte coletivo/outro & 5,3 & 6,2 & 4,8 & \\
\hline Tipo de queda & $(2255)$ & $(1401)$ & $(854)$ & \\
\hline Mesmo nível & 65,5 & 67,3 & 62,7 & 0,0000 \\
\hline Leito/mobília & 4,7 & 5,1 & 4,1 & \\
\hline Andaime/ escada/degrau & 11,2 & 8,2 & 15,8 & \\
\hline Árvore/ telhado/laje & 5,7 & 6,3 & 4,7 & \\
\hline Buraco/outros níveis & 13,0 & 13,1 & 12,7 & \\
\hline Tipo de queimadura & $(112)$ & $(46)$ & $(66)$ & \\
\hline Fogo/chama & 21,8 & 23,2 & 21,0 & 0,4609 \\
\hline Substância quente & 37,1 & 45,9 & 32,0 & \\
\hline Objeto quente & 11,7 & 10,5 & 12,5 & \\
\hline Outros $^{c}$ & 29,4 & 20,5 & 34,6 & \\
\hline Outros acidentes & $(2346)$ & $(1285)$ & $(1061)$ & \\
\hline Ferimento cortante & 19,6 & 18,0 & 21,4 & 0,0000 \\
\hline Acidentes com animais & 9,0 & 9,5 & 8,4 & \\
\hline Queda de objeto sobre a pessoa & 8,3 & 7,3 & 9,3 & \\
\hline Choque contra objeto/pessoa & 33,2 & 39,6 & 25,9 & \\
\hline Entorse/esmagamento & 17,3 & 14,7 & 20,4 & \\
\hline Outros $^{\mathrm{d}}$ & 12,7 & 11,0 & 14,6 & \\
\hline Tipo de violência & $(730)$ & $(183)$ & $(547)$ & \\
\hline Lesão autoprovocada & 9,7 & 11,6 & 9,1 & 0,3733 \\
\hline Agressão $^{e}$ & 90,3 & 88,4 & 90,9 & \\
\hline Agressor era um familiar ${ }^{f}$ & $(593)$ & $(150)$ & $(443)$ & \\
\hline Sim & 12,3 & 18,2 & 10,2 & 0,0207 \\
\hline Não & 87,8 & 81,8 & 89,8 & \\
\hline
\end{tabular}

Fonte: MS, SVS, Sistema de Vigilância de Violências e Acidentes-VIVA, Inquérito 2009.

a) Para algumas variáveis o número de atendimentos divergiu devido a dados faltantes (ignorado/em branco); b) Teste do quiquadrado; c) Inclui choque elétrico e substâncias químicas; d) Inclui sufocação/engasgamento, corpo estranho, afogamento, envenenamento/intoxicação, ferimento por arma de fogo; e) Inclui maus-tratos e intervenção por agente público legal; f) Referese somente aos casos de agressão. Inclui: pai, mãe, padrasto, madrasta, outro familiar, exceto companheiro/ex-companheiro. 


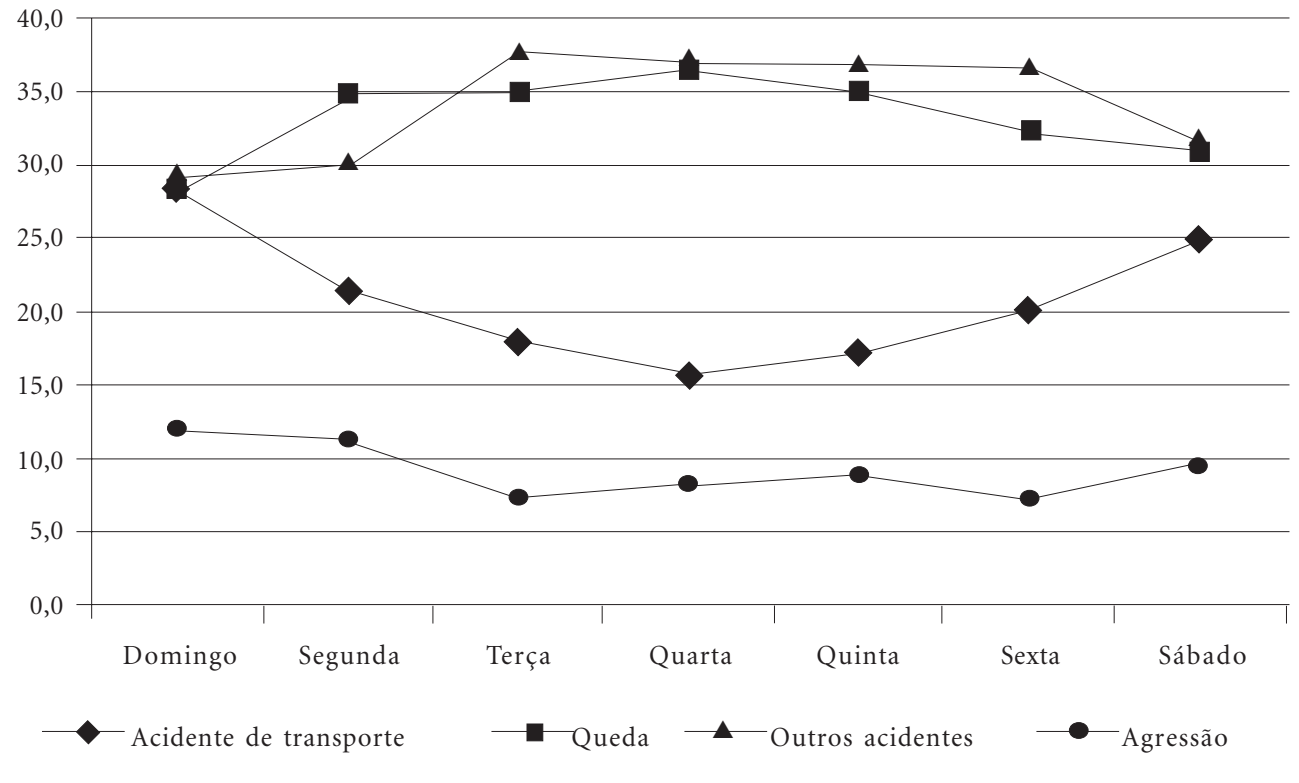

Figura 1. Distribuição dos atendimentos ${ }^{a}$ de emergência por acidentes e violência entre adolescentes, segundo o dia do atendimento, em 23 capitais e Distrito Federal, Brasil, 2009.

Fonte: MS, SVS, Sistema de Vigilância de Violências e Acidentes-VIVA, Inquérito 2009.

a) Excluídos os atendimentos por queimadura $(n=111 ; 1,6 \%)$ e lesão autoprovocada $(n=82 ; 1,0 \%)$.

entre $8 \mathrm{~h}$ e $9 \mathrm{~h}$. As quedas foram mais frequentes ao longo do período diurno e com pico entre $14 \mathrm{~h}$ e $15 \mathrm{~h}$ e de $19 \mathrm{~h}$ às $20 \mathrm{~h}$. Os outros acidentes mostraram picos às $16 \mathrm{~h}$. As agressões tiveram maior proporção de ocorrências no período noturno entre as $20 \mathrm{~h}$ e $23 \mathrm{~h}$ (Figura 2).

\section{Discussão}

As causas externas - as violências e os acidentes constituem a terceira causa de óbito no país e em algumas regiões chegam a ser a segunda, entretanto, entre adolescentes representam a primeira causa de morte ${ }^{6}$. Os dados do Inquérito VIVA 2009 vêm complementar a compreensão dessa situação, sob a perspectiva da morbidade atendida nos hospitais/serviços responsáveis pelo atendimento de emergências e porta de entrada desses casos no SUS, ao revelar outra face desse tipo de ocorrência, constituída pelos casos de baixa e média gravidade.

Dentre as principais causas de acidentes na adolescência encontram-se as quedas e os acidentes de trânsito e, entre as violências, predominam as agressões. Os adolescentes do sexo masculino sofreram mais acidentes em todas as faixas etárias; os dados demográficos aqui demonstrados corroboram estudos da literatura que apontam para o maior acometimento por causas externas no sexo masculino $0^{3,7-10,17,20}$. Este fato tem sido atribuído a questões culturais, que estimulam os meninos a realizarem atividades com maior potencial de exposição a acidentes e violências, conferem a eles maior liberdade e, por consequência, causam maior exposição a situações de risco, o que tende a se estender para a adolescência e a vida adulta ${ }^{11,21-23}$

Com relação às violências, os adolescentes do sexo masculino as sofreram mais que as meninas nas duas faixas etárias estudadas. O sexo masculino é apontado como variável preditora de comportamentos violentos quer seja em situações de conflito quer seja de vitimização ${ }^{24}$. Uma possível explicação para este fato é que o comportamento agressivo é tolerado e muitas vezes estimulado em sociedades com dominação de padrões culturais machistas. Não há apenas diferença entre sexos nas taxas de exposição à violência, as consequências também são diversas, como por exemplo, a predisposição do sexo feminino para desenvolver estresse pós-traumático e depressão 

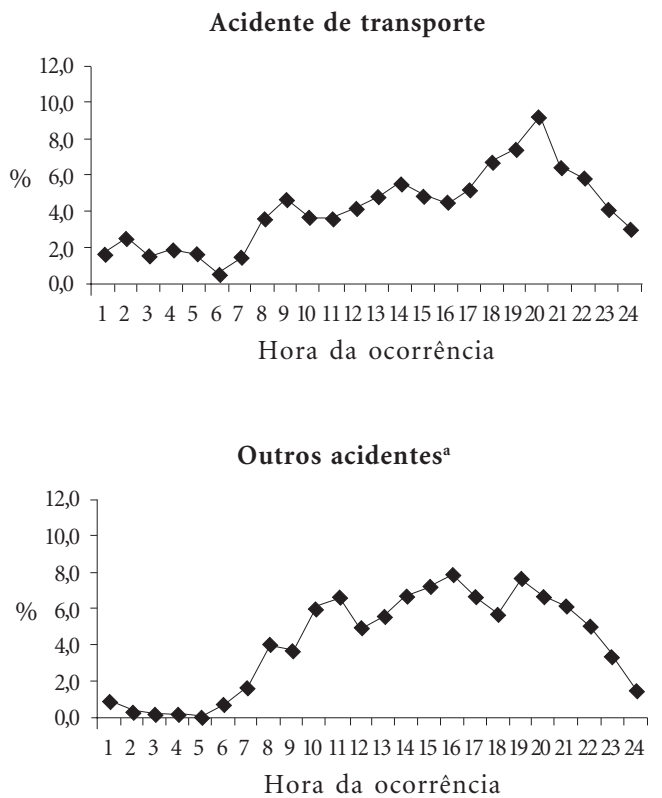
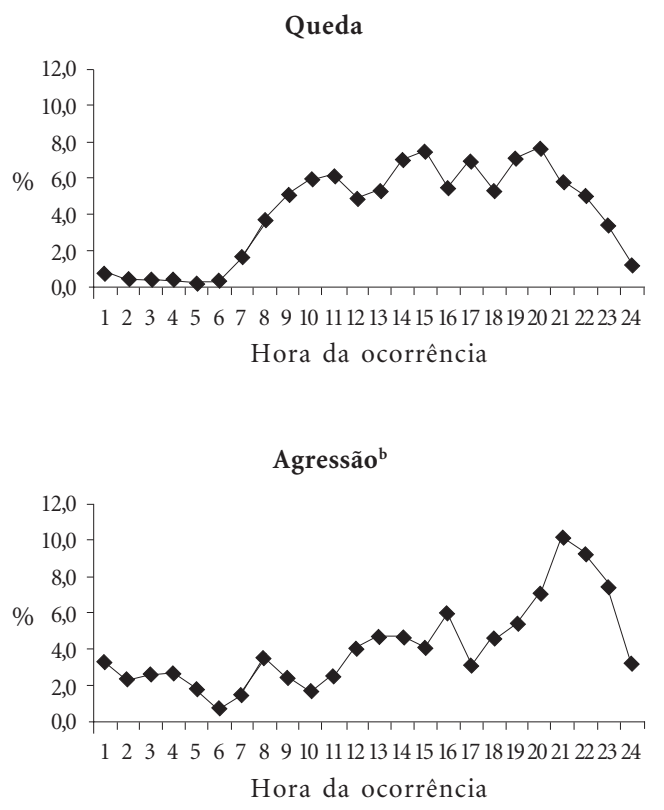

Figura 2. Distribuição dos atendimentos ${ }^{c}$ de emergência por acidentes e violência entre adolescentes, segundo hora da ocorrência, em 23 capitais e Distrito Federal, Brasil, 2009.

Fonte: MS, SVS, Sistema de Vigilância de Violências e Acidentes-VIVA, Inquérito 2009.

a) Excluídos os atendimentos por queimadura $(n=111 ; 1,6 \%)$ e lesão autoprovocada $(n=82 ; 1,0 \%)$; b) Inclui ferimento cortante, acidentes com animais, queda de objeto sobre a pessoa, choque contra objeto/pessoa, entorse, esmagamento, sufocação/ engasgamento, corpo estranho, afogamento, envenenamento/intoxicação, ferimento por arma de fogo; c) Inclui maus-tratos e intervenção por agente público legal.

após exposição a situações de violência é maior quando comparado aos meninos ${ }^{23}$.

A raça/cor não branca apresentou a maior proporção de atendimentos de causas externas. As análises do Inquérito VIVA 2009 foram realizadas agregando-se em não brancos as raças/ cor parda, preta, indígena e amarela, em função do pequeno número de registros para indígena e amarelo. A grande maioria neste campo corresponde à raça/cor negra (pardos e pretos), o que também foi identificado em outros estudos de morbi-mortalidade ${ }^{25,26}$, demandando ações de redução das iniquidades sociais. É conhecida a tendência de adolescentes negros serem mais frequentemente submetidos a grandes desigualdades sociais e à maior insegurança, estando, por isto, potencialmente, mais expostos aos riscos, especificamente às violências ${ }^{25,26}$.

Diversos estudos de mortalidade apontam que o risco de óbito por causa externa e por violência cresce em adolescentes mais velhos e adultos jovens $^{27,28}$. O Inquérito VIVA 2009 reforçou estes achados mostrando que, também nos serviços que atendem as causas externas, adolescentes mais velhos (15 a 19 anos) são mais vulneráveis à ocorrência de acidentes e violências em geral, além de estarem mais sujeitos a lesões mais graves. Os acidentes resultaram em contusão/entorse e atingiram mais os membros superiores e inferiores; a alta foi a evolução mais frequente, destacando-se maior proporção de internação hospitalar nas vítimas de violências entre 15 a 19 anos, o que demonstra maior gravidade das ocorrências nesta faixa de idade. As violências resultaram em cortes/ lacerações, e a cabeça foi a parte mais atingida. A alta foi a evolução mais prevalente e, de modo semelhante aos acidentes, verificou-se alta proporção de internação hospitalar entre 15 a 19 anos, mostrando também maior gravidade das ocorrências entre adolescentes mais velhos.

Os acidentes mais comuns foram as quedas, principalmente, entre os adolescentes de 10 a 14 anos, como descrito em outros estudos ${ }^{21,29,30}$, a maioria delas, no mesmo nível. As quedas entre 
os adolescentes estudados contabilizaram aproximadamente $33 \%$ dos atendimentos por causas externas, a maior proporção tendo sido observada entre os adolescentes mais novos. A literatura descreve perfil semelhante ${ }^{17,27,29,30}$. O Inquérito VIVA 2009 aponta ainda mudança em relação aos tipos de queda, com redução progressiva da queda do mesmo nível, típica de eventos no domicílio, para quedas de andaimes/escadas, árvores, buracos, denotando outros tipos de exposição, como riscos ambientais e exposição ocupacional, atingindo esta última em quase um quinto das ocorrências em adolescentes mais velhos.

As causas externas constituem a primeira causa de mortalidade entre adolescentes e em alguns estudos o tipo de acidente predominante foram os $\mathrm{AT}^{1,2,4,6}$. No nosso trabalho, os AT destacamse como causa importante de atendimentos de emergência por causas externas acidentais entre adolescentes, correspondendo a cerca de um quarto das ocorrências. Foi identificado por meio do Inquérito VIVA 2009 maior frequência dos AT entre adolescentes mais velhos (15 a 19 anos). Quanto ao tipo de vítima por AT entre crianças predominam os atropelamentos e os acidentes com ocupantes de veículos ${ }^{17,20,28}$ e, entre adolescentes, o inquérito analisado mostrou algo que ainda não está muito claro na literatura, o predomínio do condutor como tipo de vítima. Uma hipótese para isto é que o adolescente, principalmente do sexo masculino, assume o papel de condutor ignorando o risco e burlando a legislação. Aspectos culturais implicados na passagem do adolescente para a fase adulta podem ter relação com estas situações.

Existem ainda diferenças segundo o tipo de veículo: entre adolescentes mais jovens (10 a 14 anos) predominaram as bicicletas e, entre indivíduos de 15 a 19 anos, foi observado mais frequentemente o uso de motocicletas. Dos 468 adolescentes vítimas de AT e que eram condutores de automóvel /motocicleta, 37,6\% tinham entre 10 a 17 anos (dados não mostrados). Este fato é muito grave, pois expressa o não cumprimento da legislação brasileira, que só permite a habilitação para a direção de veículo automotor aos 18 anos de idade. Os riscos para os AT são inúmeros e dentre eles destacam-se o aumento progressivo da frota de veículos, inclusive de motocicletas, as condições das vias, o estado de manutenção dos veículos, os comportamentos de risco dos motoristas como: uso do álcool, desrespeito às leis de trânsito, não uso de equipamentos de segurança, entre outros ${ }^{20,28,31}$. Estes últimos fatores são responsáveis pela tendência em tratar os eventos no trânsito como violências, pois, na pior das hipóteses, traduzem negligência ou irresponsabilidade frente ao risco ${ }^{32}$.

Poucos estudos mensuram os fatores de risco para AT entre adolescentes e jovens no Brasil. Particularmente, a Pesquisa Nacional de Saúde dos Escolar (PeNSE) apontou fatos graves como: a) $18,5 \%$ dos adolescentes entre 13 e 15 anos de idade dirigirem veículo sem qualquer tipo de habilitação, infringindo as leis do trânsito, b) não uso de capacetes em 35\% dos adolescentes, c) não uso de cinto de segurança em $26 \%$; e d) ser transportado por alguém que ingeriu álcool em $18,7 \%^{13,20}$. Não estão claras as circunstâncias do ato de dirigir sem habilitação entre os adolescentes, mas trabalha-se com a hipótese de que algo tão grave, no mínimo, conta com a conivência dos pais ou com o não estabelecimento de limites em tal prática. Inclusive, a justiça tem imputado a responsabilidade aos pais, em casos de acidentes no trânsito praticado por menores ${ }^{32}$. Essas atitudes e práticas precoces para a idade podem trazer sérias e graves consequências, como aponta o presente estudo que contabiliza mais da metade das ocorrências como sendo devidas à prática inadequada de adolescentes condutores de motocicleta. Essas situações decorrem do fato de que, nesta faixa etária, adolescentes e jovens subestimam riscos e se julgam inatingíveis. Daí, a importância de estudos que, como este, ajudam a conhecer melhor as ocorrências e a subsidiar políticas entre educadores, pais, e apoio à legislação adequada para esta faixa.

Os outros acidentes comuns foram choque contra objetos, ferimentos cortantes e queda de objeto contra pessoa, circunstâncias as mais diversas, concordando com outros estudos ${ }^{7,21,22,30,33}$. As queimaduras ocorreram em menor proporção, menos de $2 \%$, destacando-se exposição a substâncias quentes, fogo ou chama. Resultados semelhantes foram mencionados por outros autores $^{21,34,35}$. A queimadura gera diferentes necessidades às vítimas e causa impacto em sua saúde mental, sua qualidade de vida e nas suas relações sociais bem como desconfortos físicos e limitações funcionais ${ }^{34,35}$. Apesar de pouco frequente na adolescência, as queimaduras nesta fase da vida podem acarretar danos físicos e emocionais duradouros, o que torna a sua prevenção e reabilitação ferramentas imprescindíveis.

Os locais mais frequentes de ocorrência de acidentes e violências são a rua, seguida do domicilio, o que está de acordo com outras pesquisas e mostra a importância destes espaços em ações de promoção à saúde ${ }^{5,7,14}$. A escola ocupa o terceiro lugar: 
no nosso estudo, mais de $1 / 4$ dos adolescentes sofreram acidentes na escola/prática esportiva, e este número decresceu com o aumento da idade ${ }^{8}$.

O maior número de AT e de agressões ocorreu nos finais de semana e nos horários noturnos, fortalecendo, de modo coerente com outros estudos $^{7,17,31}$, a provável associação com consumo de álcool, período da semana e horário do dia. A literatura mostra que medidas como o fechamento de bares e estabelecimentos em horários noturnos e, consequentemente, a não venda de bebidas alcoólicas, reduziram as mortes por violência em Diadema (SP), expondo a necessidade de medidas regulatórias neste sentido ${ }^{36}$. Já as quedas ocorrem mais durante os dias de semana e nos horários diurnos, manifestando uma possível relação entre ocorrências domésticas e quedas no mesmo nível ${ }^{17,29}$.

Em relação à natureza da lesão predominaram as contusões em acidentes e os cortes/lacerações/amputação nas violências. Os membros superiores também foram a parte do corpo mais atingida em acidentes e a cabeça/pescoço, nas violências ${ }^{10,21}$, as últimas podendo remeter à intencionalidade do ato, isto é, ferir gravemente ou mesmo matar. Corroborando esta afirmativa, as violências corresponderam à menor proporção de evolução para a alta e maior proporção de internação. Além disso, as frequências de internações e de atendimentos pré-hospitalares (SAMU) foram maiores na faixa etária de 15 a 19 anos e também sugerem o aumento da gravidade das ocorrências por violências entre adolescentes mais velhos. Torna-se importante analisar estas ocorrências em função da sua evitabilidade, buscando atuação integrada da sociedade, família e escola em prol da sua redução.

O Inquérito VIVA 2009 apontou que 12,3\% das violências foram praticadas por membro da família, reduzindo esta proporção com o aumento da idade dos adolescentes (15 a 19 anos). Estudos como a PENSE mostraram que cerca de 9,5\% de todos os estudantes entrevistados, independentemente do sexo, relataram agressão física cometida por um familiar. A violência familiar aponta para uma realidade preocupante, pois, neste caso, a família exerce um papel oposto ao que se espera e acaba sendo fonte de violência e agressão ${ }^{5}$. Os direitos das crianças e adolescentes estão bem fundamentados no Estatuto da Criança e Adolescente (ECA) em vigor desde 1990, onde é assegurado que nenhuma criança ou adolescente deve ser objeto de discriminação, negligência, exploração, violência, crueldade ou agressão dentro ou fora da família ${ }^{37}$.
Dentre os limites do estudo, citam-se a inclusão apenas de serviços selecionados do SUS (não inclusão de atendimentos do setor privado e das vítimas que não procuraram o serviço de urgência/emergência do SUS) e o uso da base de dados de serviços sentinelas do SUS, não permitindo estimativas populacionais. Entretanto, este tipo de inquérito apresenta vantagens pela maior sensibilização das equipes, articulação com a rede de serviços de proteção à vítima de violência, além de possibilitar o uso das informações para o planejamento. Outro limite consiste na própria dificuldade da coleta e no momento em que ela ocorre, que pode levar a omissão de informações sobre as circunstâncias, em especial nos casos decorrentes de violências.

Por outro lado, é fundamental conhecer as lesões de menor gravidade, as quais não levam à morte ou internação, mas são responsáveis por alta demanda nas unidades de emergência e podem resultar em marcas profundas para milhares de adolescentes. Os dados do Inquérito VIVA 2009 apoiam o planejamento de políticas públicas (micro e as macro-políticas), buscando maior efetividade da atenção à saúde da criança e do adolescente ${ }^{5,17,38}$.

O Inquérito VIVA 2009 mostra, ainda, a vulnerabilidade a que muitos adolescentes estão expostos, revelando a necessidade de tanto se promover ambientes como se adotar comportamentos seguros e saudáveis, oferecer o acompanhamento das famílias e dos adolescentes vulneráveis e garantir o atendimento voltado à recuperação e à reabilitação. Estes dados reforçam a necessidade de avançar na capacitação de recursos humanos para a identificação, a notificação, o encaminhamento e a atenção aos casos de acidentes e violências, bem como na atuação interdisciplinar e intersetorial às vítimas, contemplando uma postura acolhedora, escuta atenta, a responsabilização e o vínculo ${ }^{5,17,38}$.

A redução do número de acidentes e violências no país e, consequentemente, de mortes, lesões, hospitalizações e incapacidades, ainda representa um desafio para a sociedade. Para tanto, processos indutores e fomentadores, incluindo medidas educativas, aprimoramento e implementação da legislação adequada, venda de bebidas alcoólicas a menores, horário de fechamento noturno de estabelecimentos, fiscalização no trânsito, proibição de propagandas de bebida alcoólica, vigilância e funcionamento do Conselho Tutelar, são fundamentais e devem ser ampliados para toda a população $0^{6,18,31,39}$. Conhecer a realidade epidemiológica dos acidentes e violências, incluindo as informações 
sobre as vítimas e a magnitude e a distribuição dos fatores de risco, representa uma importante ferramenta para as políticas de prevenção, promoção à saúde e da cultura de paz, visando à redução da morbimortalidade ${ }^{3}$. Além disso, a abordagem de grupos estratégicos como o dos adolescentes indica ser oportuna para o desenvolvimento de uma cultura de paz no Brasil ${ }^{18,20}$.

\section{Colaboradores}

DC Malta responsável pela concepção do projeto, delineamento do estudo, revisão da literatura e elaboração da versão inicial do texto. MDM Mascarenhas e RTI Bernal foram responsáveis pelas análises estatísticas, análise e interpretação dos dados e contribuíram na revisão crítica do artigo. SSCA Andrade, ACM Neves, EM Melo e JB Silva Junior participaram da análise e interpretação dos dados e contribuíram na revisão crítica do artigo. Todos os autores aprovaram a versão final do manuscrito. 


\section{Referências}

1. World Health Organization (WHO). Health topics: adolescent health. Geneva: WHO; 2010. [acessado 2010 jan 30]. Disponível em: http://www.who.int/ topics/adolescent_health/en/.

2. World Health Organization (WHO). World report on child injury prevention. Geneva: WHO/Unicef; 2008.

3. World Health Organization (WHO). World report on violence and health. Geneva: WHO; 2002.

4. World Health Organization (WHO). World report on road traffic injury prevention. Geneva: WHO; 2004.

5. Malta DC, Sardinha LMV, Mendes I, Barreto SM, Giatti L, Castro IRR, Moura L, Dias AJR, Crespo C. Vivência de violência entre escolares brasileiros: resultados da Pesquisa Nacional de Saúde do Escolar (PeNSE). Cien Saude Colet 2010; 15(2):3053-3063.

6. Brasil. Ministério da Saúde (MS). Secretaria de Vigilância em Saúde. Departamento de Análise de Situação em Saúde. Saúde Brasil 2010: uma análise da situação de saúde. Brasília: MS; 2011.

7. Gaspar VLV, Lamounier JA, Cunha FMA, Gaspar JC. Fatores relacionados a hospitalizações por injúrias em crianças e adolescentes. J. Pediatr. 2004; 80(6):447-452.

8. Chen G, Smith GA, Deng S, Hostetler SG, Xiang H. Nonfatal injuries among middle-school and highschool students in Guangxi, China. Am J Public Health 2005; 95(11):1989-1995.

9. Gawryszewski VP, Rodrigues EMS. O impacto das causas externas de morbidades no Brasil, 2003. Sao Paulo Med. J. 2006; 124(4):208-213.

10. Franciozi CES, Tamaoki MJS, Araújo EFA, Dobashi ET, Utumi CE, Pinto JA, Ishida A. Trauma na infância e adolescência: epidemiologia, tratamento e aspectos econômicos em um hospital público. Acta ortop. bras. 2008; 16(5):261-265.

11. Minayo MCS. Violência e Saúde. Rio de Janeiro: Editora Fiocruz; 2006.

12. Brasil. Instituto Brasileiro de Geografia e Estatística (IBGE). Pesquisa Nacional de Saúde do Escolar 2009. Rio de Janeiro: IBGE; 2009. [acessado 2010 ago 20]. Disponível em: <http://www.ibge.gov.br/home/estatistica/populacao/pense/pense.pdf $>$.

13. Minayo MCS. Pesquisa social: teoria, método e criatividade. 19a Edição. Petropólis: Vozes; 2001.

14. Mascarenhas MDM, Malta DC, Silva MMA, Lima CM, Carvalho MGO, Oliveira VLA. Violência contra a criança: revelando o perfil dos atendimentos em serviços de emergência, Brasil, 2006 e 2007. Cad Saude Publica 2010; 26(2):347-357.

15. Stoddard SA, Henley SJ, Sieying RE, Bolland J. Social connections, trajectories of hopelessness and serious violence in impoverished urban youth. J. Youth Adolesc 2011; 40(3):278-295.

16. Zaniol E, Dal Molin F, Andreoli G. Revista do Departamento de Psicologia - UFF 2007; 19(1):243-252.

17. Vendruscolo TS, Ferriani MGC, Silva MAI. Public care policies for child and adolescent victims of domestic violence. Revista Latino Am Enf 2007; 15(N. Esp.):812-819.
18. Brasil. Ministério da Saúde (MS). Secretaria de Vigilância em Saúde. Departamento de Análise de Situação de Saúde. VIVA: vigilância de violências e acidentes, 2008 e 2009. Brasília: MS; 2010.

19. Brasil. Ministério da Saúde. Conselho Nacional de Saúde. Resolução no . 196/1996. Diretrizes e Normas Regulamentadoras de Pesquisas Envolvendo Seres Humanos. Diário Oficial da União 1996; out 10.

20. Morais Neto OL, Malta DC, Mascarenhas MDM, Duarte EC, Silva MMA, Oliveira KB, Lima CM, Porto, DL. Fatores de risco para acidentes de transporte terrestre entre adolescentes no Brasil: Pesquisa Nacional de Saúde do Escolar (PeNSE). Cien Saude Colet 2010; 15(2):3043-3052.

21. Martins CBG, Andrade SM. Epidemiologia dos acidentes e violências entre menores de 15 anos em município da região sul do Brasil. Revista Latino Am Enf 2005; 13(4):530-537.

22. Cavalcanti AL, Martins VM, Lucena RN, GranvilleGarcia AF, Menezes VA. Morbidade por causas externas em crianças e adolescentes em Campina Grande, Paraíba. Arq Catarin Med 2008; 37(3):27-33.

23. Hanson RF, Borntrager C, Self-brown S, Kilpatrick DG, Saunders B, Resnick HS, Amstadter A. Relations among Gender, Violence Exposure, and Mental Health: The National Survey of Adolescents I. Am J Orthopsychiatry 2008; 78(3):313-321.

24. Aceves MJ, Hinshaw SP, Mendoza-Denton R, PageGould E. J. Youth Adolescence 2010; 39:658-669.

25. Brasil. Ministério da Saúde (MS). Secretaria de Vigilância em Saúde. Departamento de Análise de Situação em Saúde. Saúde Brasil 2007: uma análise da situação de saúde. Brasília: MS; 2008.

26. Araújo EM, Costa MCN, Hogan VK, Mota ELA, Araújo TM, Oliveira NF. Diferenciais de raça/cor da pele em anos potenciais de vida perdidos por causas externas. Rev Saude Publica 2009; 43(3):405-412.

27. Barros MDA, Ximenes R, Lima MLC. Mortalidade por causas externas em crianças e adolescentes: tendências de 1979 a 1995. Rev Saude Publica 2001; 35(2):142-149.

28. Reichenheim ME, Souza ER, Moraes CL, MelloJorge MHP, Silva CMFP, Minayo MCS. Violência e lesões no Brasil: efeitos, avanços alcançados e desafios futuros. The Lancet 2011; 75-89. [acessado 2012 fev 24]. Disponível em: http://download.thelancet. com/flatcontentassets/pdfs/brazil/brazilpor5.pdf

29. Martins CBG, Andrade SM. Estudo descritivo de quedas entre menores de 15 anos no município de Londrina (PR, Brasil). Cien Saude Colet 2010; 15(2): 3167-3173.

30. Martins CBG. Acidentes na infância e adolescência: uma revisão bibliográfica. Rev Bras Enferm 2006; 59(3):344-348.

31. Mascarenhas MDM, Malta DC, Silva MMA, Carvalho CG, Monteiro RA, Morais Neto OL. Consumo de álcool entre vítimas de acidentes e violências atendidas em serviços de emergência no Brasil, 2006 e 2007. Cien Saude Colet 2009; 14(5):1789-1796. 
32. Brasil. Ministério da Justiça (MJ). Lei 10.406 de 10 de janeiro de 2002. Código Civil Brasileiro. Brasília: MJ; 2002.

33. Brasil. Ministério da Saúde (MS). Impacto da violência na saúde dos brasileiros. Brasília: MS; 2005.

34. Martins CBG, Andrade SM. Acidentes com corpo estranho em menores de 15 anos: análise epidemiológica dos atendimentos em pronto-socorro, internações e óbitos. Cad Saude Publica 2008; 24(9):19831990.

35. Montes SF, Barbosa MH, Sousa Neto AL. Aspectos clínicos e epidemiológicos de pacientes queimados internados em um Hospital de Ensino. Rev esc enferm USP 2011; 45(2):369-373.

36. Costa MCS, Rossi LA, Lopes LM, Cioffi CL. Significados de qualidade de vida: análise interpretativa baseada na experiência de pessoas em reabilitação de queimaduras. Revista Latino Am Enf 2008; 16(2): 252-259.

37. Duailibi S, Ponicki W, Pinsky I, Laranjeira R, Raw M. The effect of restricting opening hours on alcohol related violence. Am J Public Health 2007; 97(12): 2276- 2280.

38. Brasil. Lei $\mathrm{n}^{\circ} 8.069$, de 13 de julho de 1990. Dispõe sobre o Estatuto da Criança e do Adolescente, e dá outras providências. Diário Oficial da União 1990; 16 jul.

39. Gawryszewski VP, Silva MMA, Malta DC, Mascarenhas MDM, Costa VC, Matos SG, Moraes Neto OL, Monteiro RA, Carvalho CG. Magalhães ML. A proposta da rede de serviços sentinela como estratégia da vigilância de violências e acidentes. Cien Saude Colet 2007; 11(Supl.):1269-1278.

Artigo apresentado em 10/06/2012

Aprovado em 04/07/2012

Versão final apresentada em 14/07/2012 